\title{
Energy decay rates and convexity for hyperbolic equations with nonlinear dissipation
}

\author{
Fatiha Alabau-Boussouira*t \\ Universite de Metz, LMAM UMR 7122 et INRIA, Projet CORIDA \\ Ile du Saulcy, 57045 METZ Cedex 01, FRANCE \\ E-mail: alabau@univ-metz.fr
}

In this paper, we consider hyperbolic systems subjected to nonlinear dissipation without specifying the growth of this dissipation around the origin. It is well-known that this growth determines the behavior of the energy of the solutions at time goes to infinity. We first situate the problem in the literature and show how convexity arguments, used in a sharp way, together with weighted integral inequalities allow us to obtain a general semi-explicit energy decay formula. We will illustrate these results on several examples and give optimality results for some of them.

Control Systems: Theory, Numerics and Applications

30 March - 1 April 2005

Rome

\footnotetext{
${ }^{*}$ Speaker.

${ }^{\dagger}$ The speaker thanks INDAM for their kind invitation.
} 


\section{Introduction}

Consider the following model problem:

$$
\left\{\begin{array}{l}
u_{t t}-\Delta u+\rho\left(u_{t}\right)=0 \text { on }(0,+\infty) \times \Omega \\
u=0 \text { on }(0,+\infty) \times \Gamma \\
u(0, .)=u^{0}(.), u_{t}(0, .)=u^{1}(.) \text { on } \Omega
\end{array}\right.
$$

Here, we assume that $\Omega$ is a non-empty bounded open set in $\mathbb{R}^{N}$ having a boundary $\Gamma$ of class $C^{2}$. If $\rho$ is assumed to have a linear growth close to zero (and linear growth at infi nity but for different reasons), it is well-known that the energy decays exponentially as times goes to infi nity. Results for nonlinear dissipative equations with polynomial growth of the feedback at the origin, can be found in [21], [15], [8] and the references therein. These results, in fi nal form, state that if $\rho$ satisfi es for some $p>1$ :

$$
c_{1}|v|^{p} \leq|\rho(v)| \leq c_{2}|v|^{1 / p} \text { for }|v| \leq 1,
$$

then the energy of solutions has the decay rate

$$
E(t) \leq C(E(0)) t^{-2 /(p-1)} \quad \forall t>0,
$$

where $C(E(0))$ stands for a constant which depends continuously on $E(0)$.

For decay rates in the case where no growth condition on the dissipation term at the origin is assigned a priori, we refer to [9], [13], ( see also [5], [12]) and to [1] ([2]) for a discussion on the subject. For a further large list of references on this question, we also refer the interested reader to [10].

Many other results for dissipative hyperbolic equations are available, so the above list has no pretention to be exhaustive, but it is limited for size restrictions. Let us now consider a specific example, to illustrate our purpose. For this, we consider a function $\rho$ as follows

$$
\text { Example }\left\{\begin{array}{l}
\rho \text { is a strictly increasing odd function } \in C^{1}(\mathbb{R}) \text { such that } \\
|v| \leq|\rho(v)| \leq C|v|, \text { if }|v| \geq 1 \\
\rho(|v|)=e^{-\left(\ln \left(\frac{1}{|v|}\right)\right)^{p}}, \quad \text { if }|v| \leq r_{0}
\end{array}\right.
$$

where $1<p<2$ and where $r_{0}$ is a given suffi ciently small positive number.

The energy $E$ of a solution $u$ is given by

$$
E(t)=\frac{1}{2} \int_{\Omega}\left(u^{\prime 2}+|\nabla u|^{2}\right) .
$$

The dissipation term $\rho\left(u_{t}\right)$ induces a dissipation of the energy. This means that one can prove that the energy of strong solutions satisfi es the following dissipation relation

$$
E^{\prime}(t)=-\int_{\Omega} u_{t} \rho\left(u_{t}\right) d x
$$

For this example, one can remark that the function $\rho$ goes to zero faster than any polynomial as its argument goes to zero. So, the dissipation is very weak close to the origin. Several questions 
arise. Is this weak dissipation suffi cient to induce decay of the energy as times goes to infi nity? If so, is it possible to obtain precise decay rates of the energy for arbitrary growth assumptions of the dissipation term close to the origin? Can one prove that these decay rates are optimal? And fi nally, is it possible to fi nd a general method, valid for abstract hyperbolic equations and applicable for elasticity, Petrowsky equations, Timoshenko beams..., valid for localized as well as boundary damping, and for arbitrary dissipation terms?

A positive general answer can be given to the above questions, with limitations to the onedimensional wave equation with boundary damping for the proof of optimality. Let us fi rst give an idea of the possible results for the above model example. We prove

Theorem 1. Assume that $\Omega, \Gamma$, and $\rho$ are given as above. Then the energy of solution of (1.1) satisfies for the above example, the estimate

$$
E(t) \leq \beta_{E(0)}\left(\Theta^{-1}\left(\frac{D}{t}\right)\right)^{2}
$$

for $t$ sufficiently large and for all $\left(u_{0}, u_{1}\right) \in H_{0}^{1}(\Omega) \times L^{2}(\Omega)$, where $\beta_{E(0)}$ is a constant which depends continuously on $E(0)$ and is of the form:

$$
\beta_{E(0)}=\max \left(\eta_{1}, \eta_{2} E(0)\right),
$$

where $\eta_{1}, \eta_{2}$ and $D$ are three positive constants which do not depend on $E(0)$, and where the function $\Theta$ is defined by:

$$
\Theta(x)=x^{-1}\left(\ln \left(\frac{1}{x}\right)\right)^{p-1} e^{-\left(\ln \left(\frac{1}{x}\right)\right)^{p}} \quad x \in(0,1) .
$$

Similar results can be proved for more general hyperbolic equations, locally distributed dissipation (with localisation conditions), boundary dissipation. The purpose of this paper is to give some ideas of the important properties for the abstract equations and then of how one can apply these results to concrete examples.

Our method is based on weighted integral inequalities for the energy. The choice of the weight is crucial. Indeed, let us denote by $O$ the active region on which the dissipation is effective. Then, the linear kinetic energy of the solution is given by:

$$
\int_{O}\left|u_{t}\right|^{2} d x
$$

whereas the nonlinear kinetic energy of the solution is given by:

$$
\int_{\Omega}\left|\rho\left(u_{t}\right)\right|^{2} d x
$$

To choose the weight, we point out the link that exists between dissipation of the energy and the linear and nonlinear kinetic energy. This link is made through convexity of an explicit function connected to the growth of the feedback at the origin, and requires the use of Jensen, and Young's inequalities for convex proper functions and their convex conjugate. 


\section{Main results}

For size constraints and the clarity of the exposition, we will only state the main results concerning the locally distributed case. We refer to [1] for complete results.

Let $\Omega$ be a bounded open subset of $\mathbb{R}^{N}$ with a smooth boundary denoted by $\Gamma$ and $\omega$ be an open subset of $\Omega$ of positive measure.

We set $H=L^{2}(\Omega)$ and denotes by $|\cdot|_{H}$ the $L^{2}$-norm on $\Omega$ in all what follows. Let $A$ be a coercive selfadjoint densely defi ned linear unbounded operator in $H$, with domain $D(A)$. The above abstract equation can as well represent Petrowsky equation or the system of linear elasticity. We set $V=D\left(A^{1 / 2}\right)$. The operator $B$ is the operator defi ned from $\bar{\Omega} \times H$ on $H$, by

$$
B(., v)=\rho(., v) .
$$

We assume that the dissipation term $\rho$ satisfi es the following hypotheses:

$$
(H F)\left\{\begin{array}{l}
\rho \in C(\bar{\Omega} \times \mathbb{R}) \text { and is monotone increasing with respect to the second variable } \\
\exists a \in C(\bar{\Omega}), a \geq 0 \text { on } \Omega \text { and a strictly increasing odd function } g \in C^{1}(\mathbb{R}) \text { such that } \\
a(x)|v| \leq|\rho(x, v)| \leq C a(x)|v|, \quad \forall x \in \Omega, \text { if }|v| \geq 1 \\
a(x) g(|v|) \leq|\rho(x, v)| \leq C a(x) g^{-1}(|v|), \quad \forall x \in \Omega, \text { if }|v| \leq 1, \\
a(x) \geq a_{-}>0, \quad \forall x \in \omega, \\
\exists r_{0} \in(0,1), g\left(r_{0}\right)<1 \text { such that the function } \sqrt{\cdot} g(\sqrt{\cdot}) \text { is strictly convex on }\left[0, r_{0}^{2}\right] \\
\text { and } g \in C^{2}\left(\left[0, r_{0}\right]\right), g^{\prime}(0)=0 .
\end{array}\right.
$$

where $g^{-1}$ denotes the inverse function of $g$ and where $C$ is a positive constant. Then, thanks to hypothesis $(H F), B$ is a monotone continuous operator defi ned from $\bar{\Omega} \times H$ on $H$.

Remark: If $g^{\prime}(0) \neq 0$, then $g$ has a linear growth at the origin. In this case, it is well-known that the energy of the system decays exponentially.

We consider the following second order equation:

$$
\begin{array}{r}
u^{\prime \prime}(t)(.)+A u(t)(.)+B\left(., u^{\prime}(t)(.)\right)=0 \quad t>0, \\
u(0)(.)=u^{0}(.), u^{\prime}(.)=u^{1}(.),
\end{array}
$$

where We recall the following existence and regularity result (see e.g. [6] for the proof) using the theory of maximal nonlinear monotone operator:

Proposition 2. Assume hypothesis $(H F)$. Then for all $\left(u^{0}, u^{1}\right) \in V \times H$, the problem (2.2) has a unique solution $u \in \mathcal{C}([0,+\infty) ; V) \times \mathcal{C}^{1}([0,+\infty) ; H)$. Moreover, for all $\left(u^{0}, u^{1}\right) \in D(A) \times V$, the solution of $(2.2)$ is in $L^{\infty}([0,+\infty) ; D(A)) \times W^{1, \infty}([0,+\infty) ; V) \times W^{2, \infty}([0,+\infty) ; H)$ and its energy defined by:

$$
E(t)=\frac{1}{2}\left(\left|u^{\prime}(t)\right|_{H}^{2}+\left|A^{1 / 2} u(t)\right|_{H}^{2}\right)
$$

satisfies the following dissipation relation:

$$
E^{\prime}(t)=-\int_{\Omega} u^{\prime}(t)(x) \rho\left(x, u^{\prime}(t)(x) d x \leq 0 .\right.
$$


We need to introduce some preliminary defi nitions.

Assume hypothesis (HF). We fi rst defi ne a function $H$ by

$$
H(x)=\sqrt{x} g(\sqrt{x})
$$

We then defi ne a function $\widehat{H}$ by

$$
\widehat{H}(x)= \begin{cases}H(x) & , \text { if } x \in\left[0, r_{0}^{2}\right], \\ +\infty & , \text { if } x \in \mathbb{R} \backslash\left[0, r_{0}^{2}\right],\end{cases}
$$

We consider the function $F$ given by:

$$
F(y)= \begin{cases}\frac{\widehat{H}^{\star}(y)}{y} \quad, \text { if } y \in(0,+\infty), \\ 0 \quad \text { if } y=0\end{cases}
$$

where $\widehat{H}^{\star}$ stands for the convex conjugate function of $\widehat{H}$, that is

$$
\widehat{H}^{\star}(y)=\sup _{x \in \mathbb{R}}\{x y-\widehat{H}(x)\} .
$$

Then $F$ is a strictly increasing continuous onto function from $[0,+\infty)$ on $\left[0, r_{0}^{2}\right)$ (see [1] for more details). We now defi ne a function $f$ from $\left[0,2 \beta r_{0}^{2}\right)$ onto $[0,+\infty)$ by

$$
f(s)=F^{-1}\left(\frac{s}{2 \beta}\right) \quad \forall s \in\left[0,2 \beta r_{0}^{2}\right) .
$$

where $\beta=\beta_{E(0)}$ is given by

$$
\beta_{E(0)}=\max \left(\eta_{1}, \eta_{2} E(0)\right)
$$

where $\eta_{1}$ and $\eta_{2}$ do not depend on $E(0)$. Then, one can easily show that $f$ is a nonnegative $C^{1}$ and strictly increasing function defi ned from $\left[0,2 \beta r_{0}^{2}\right)$ onto $[0,+\infty)$ (see [1] for the proof). For $r_{0}$ as in hypothesis (HF) and $\beta=\beta_{E(0)}$ defi ned as above, we set

$$
\begin{array}{r}
\mathcal{F}=\mathcal{F}_{\beta_{E(0)}, r_{0}^{2}}=\left\{f \in C^{1}\left(\left[0,2 \beta_{E(0)} r_{0}^{2}\right),[0, \infty)\right),\right. \\
f \text { strictly increasing, nonnegative and onto }\} .
\end{array}
$$

We prove the following key result for obtention of energy decay rates:

Theorem 3. Assume hypothesis $(H F)$. Let $\delta_{i}>0$ for $i=1,2,3$ and $\left(u^{0}, u^{1}\right) \in V \times H$, satisfying $0<\left|u^{1}\right|_{H}^{2}+\left|A^{1 / 2} u^{0}\right|_{H}^{2}$ be given and $f$ be defined as in (2.8) We assume that the energy $E$ defined by (2.3) associated to the solution of (2.2) satisfies

$$
\begin{array}{r}
\int_{S}^{T} f(E(t)) E(t) d t \leq \delta_{1} E(S) f(E(S))+\delta_{2} \int_{S}^{T} f(E(t))\left(\int_{\Omega}\left|\rho\left(x, u^{\prime}(t)(x)\right)\right|^{2} d x\right) d t+ \\
\delta_{3} \int_{S}^{T} f(E(t))\left(\int_{\omega}\left|u^{\prime}(t)(x)\right|^{2} d x\right) d t .
\end{array}
$$


Then E satisfies the estimate:

$$
E(t) \leq 2 \beta_{E(0)} z^{2}(t) \frac{z(t) g^{\prime}(z(t))-g(z(t))}{z(t) g^{\prime}(z(t))+g(z(t))}, \quad \forall t \geq \frac{T_{0}}{H^{\prime}\left(r_{0}^{2}\right)},
$$

where

$$
z(t)=\phi^{-1}\left(\frac{t}{T_{0}}\right)
$$

Here, $\phi$ is the strictly decreasing and onto function defined from $\left(0, r_{0}\right]$ on $\left[\frac{1}{H^{\prime}\left(r_{0}^{2}\right)},+\infty\right)$ by:

$$
\phi(v)=\frac{2 v}{v g^{\prime}(v)+g(v)}+4 \alpha(v),
$$

where $\alpha$ is defined on $\left(0, r_{0}\right]$ by the following integral expression

$$
\alpha(\tau)=\int_{\tau}^{r_{0}} \frac{g(u)\left(u^{2} g^{\prime \prime}(u)+u g^{\prime}(u)-g(u)\right)}{\left(u g^{\prime}(u)+g(u)\right)^{2}\left(u g^{\prime}(u)-g(u)\right)} d u
$$

Remarks: Estimates such as (2.11) are obtained using the multiplier method combined eventually with the sharp trace regularity method [10].

The terms

$$
\int_{\Omega}\left|\rho\left(x, u^{\prime}(t)(x)\right)\right|^{2} d x d t \text { and } \int_{\omega}\left|u^{\prime}(t)(x)\right|^{2} d x d t
$$

are respectively the nonlinear and linear kinetic energy of the corresponding solution. Hence, the previous theorem tells that if one can control a weighted $L^{1}$-norm of the energy in terms of weighted norms of the linear and nonlinear kinetic energy, then one can deduce the rate at which the energy decays.

We did not consider in the previous results the trivial case for which $E(0)=0$. Indeed, if $\left(u_{0}, u_{1}\right)=(0,0)$, then $E(t)=0$ for all $t \geq 0$, thanks to the decay of $E$.

\section{Applications to hyperbolic equations}

To prove the desired decay of the energy, we will apply Theorem 3. For this, we need to prove an estimate of the form (2.11). We choose to apply this result to the wave equation. But any other equation for which a multiplier method works for the linear case could be treated in a similar way. The estimate (2.11) will be obtained by the piecewise multiplier method. This method has been initiated by K. Liu [11] (for observability results). Then, Martinez [13] has developped this method for stabilization results for the wave equation.

Let $\Omega$ be a bounded open subset of $\mathbb{R}^{N}$ with a smooth boundary denoted by $\Gamma$. We assume that $\omega$ is an open subset of $\Omega$ of positive measure. We consider the following wave equation with nonlinear damping $\rho\left(., u_{t}\right)$ :

$$
\left\{\begin{array}{l}
\partial_{t t} u-\Delta u+\rho\left(x, u_{t}\right)=0 \quad \text { in } \quad \Omega \times \mathbb{R} \\
u=0 \quad \text { on } \quad \Sigma=\Gamma \times \mathbb{R} \\
\left(u, \partial_{t} u\right)(0)=\left(u^{0}, u^{1}\right) \quad \text { on } \quad \Omega
\end{array}\right.
$$


Hence, $u$ is a solution of an equation of the form (2.2), where $A$ is given by $D(A)=H^{2}(\Omega) \cap H_{0}^{1}(\Omega)$, $A=-\Delta$ and $B(., v)=\rho(., v)$. Here $D\left(A^{1 / 2}\right)=H_{0}^{1}(\Omega)$.

We defi ne the energy of a solution by

$$
E(t)=\frac{1}{2}\left(\int_{\Omega}\left|u_{t}\right|^{2}+|\nabla u|^{2}\right) d x
$$

We make the assumption $(H F)$ on the feedback. With further geometric assumptions on the active region $\omega$, stabilization (even in the linear case) holds (see [3]). We consider here the geometric assumptions which guarantee that a multiplier type method works. We refer the readers to [3] for a microlocal analysis in the linear case and to [9] for sharp trace regularity results which in both cases lead to much sharper and more general geometric conditions than the multiplier method.

We use the following notations. If $\Omega_{j} \subset \Omega$ is a Lipschitz domain, we denote by $\Gamma_{j}$ its boundary and by $v_{j}$ the outward unit normal to $\Gamma_{j}$. Moreover, if $U$ is a subset of $\mathbb{R}^{N}$ and $x \in R^{N}$, we set $d(x, U)=\inf _{y \in U}|x-y|$, and $\mathcal{N}_{\varepsilon}(U)=\left\{x \in \mathbb{R}^{N}, d(x, U) \leq \varepsilon\right\}$.

We make the following geometric assumptions on $\Omega$ and $\omega$ as in [11] and [13] (for use of the piecewise multiplier method):

$(H G)\left\{\begin{array}{l}\exists \varepsilon>0, \text { domains } \Omega_{j} \subset \Omega \text { with Lipschitz boundary } \Gamma_{j} \text { for } 1 \leq j \leq J \text { and points } x_{j} \text { in } \mathbb{R}^{N} \\ \text { such that } \Omega_{i} \cap \Omega_{j}=\emptyset \text { if } i \neq j \\ \Omega \cap \mathcal{N}_{\varepsilon}\left[\cup_{j} \gamma_{j}\left(x_{j}\right) \cup\left(\Omega \backslash \cup_{j} \Omega_{j}\right)\right] \subset \omega,\end{array}\right.$

where $\gamma_{j}\left(x_{j}\right)=\left\{x \in \Gamma_{j},\left(x-x_{j}\right) \cdot v_{j}(x)>0\right\}$. One can remark that these assumptions are a generalization of Zuazua's assumptions in [20], where he proved stabilization of a semilinear wave equation by a damping locally distributed on a set $\omega$ provided that this set contains a neighbourhood of $\left\{x \in \partial \Omega,\left(x-x_{0}\right) \cdot v>0\right\}, x_{0}$ being a fi xed point in $\mathbb{R}^{N}$. In this latter case, one has a single domain $\Omega_{1}=\Omega$ and $v$ is the unit outward normal to $\Gamma$. Then, we prove:

Theorem 4. Assume the above hypotheses $(H F),(H G)$ and that $\Omega$ is of class $C^{2}$. Let $\left(u_{0}, u_{1}\right) \in$ $H_{0}^{1}(\Omega) \times L^{2}(\Omega)$ be given, and let $f$ be an arbitrary function in the set $\mathcal{F}_{\beta_{E(0)}, r_{0}^{2}}$ defined by (2.10). Then, there exist positive contants $\delta_{i}$ for $i=1, \ldots, 4$, independent on $f$ and on $\left(u_{0}, u_{1}\right)$ such that the energy of the solutions of (3.1) satisfies the estimate (2.11).

Using this result with Theorem 3, we deduce the following corollary.

Corollary 5. Assume the above hypotheses of Theorem 4. Let $\left(u_{0}, u_{1}\right) \in H_{0}^{1}(\Omega) \times L^{2}(\Omega)$ be given. Then the energy of the solutions of (3.1) satisfies the decay estimate (2.12) for all $\left(u_{0}, u_{1}\right) \in$ $H_{0}^{1}(\Omega) \times L^{2}(\Omega)$, where $z$ is given by (2.13), $\phi$ and $\alpha$ are respectively given by (2.14) and (2.15), and where $T_{0}$ does not depend on $\left(u_{0}, u_{1}\right)$.

\section{Examples of energy decay rates}

We consider fi ve representative examples of different behavior of the dissipation term close to zero, from polynomial up to exponential. We show, how the general formula (2.12)-(2.15) leads to 
precise decay rates in a unifi ed way. We refer to [1] for comparison with the decay rates obtained in the literature.

In all what follows, we just give the expression of the function $g$ on an interval of the form $\left(0, r_{0}\right]$ where $r_{0}>0$ is chosen suffi ciently small so that the hypotheses of Theorem 4 hold. The function $g$ is then suitably extended to $\mathbb{R}$.

Theorem 6. We assume the above geometric hypotheses and that the function $g$ is given by the expressions below on an interval $\left(0, r_{0}\right]$ where $r_{0}>0$ is chosen sufficiently small so that the hypotheses of Theorem 4 hold. The function $g$ is then suitably extended to $\mathbb{R}$ (as a strictly increasing, odd, $C^{1}$ function with linear growth at $\infty$ ). Then, we have the following results:

Example 1: let $g$ be given by $g(x)=x^{p}$ where $p>1$ on $\left(0, r_{0}\right]$.

Then the energy of solution of (3.1) satisfies the estimate

$$
E(t) \leq \beta_{E(0)} t^{\frac{-2}{p-1}},
$$

for $t$ sufficiently large and for all $\left(u_{0}, u_{1}\right) \in H_{0}^{1}(\Omega) \times L^{2}(\Omega)$ and where $\beta_{E(0)}$ is defined as in (2.9).

Example 2: let $g$ be given by $g(x)=e^{-\frac{1}{x}}$ on $\left(0, r_{0}\right]$.

Then the energy of solution of (3.1) satisfies the estimate

$$
E(t) \leq \beta_{E(0)}\left(\Theta^{-1}\left(\frac{D}{t}\right)\right)^{2},
$$

for $t$ sufficiently large and for all $\left(u_{0}, u_{1}\right) \in H_{0}^{1}(\Omega) \times L^{2}(\Omega)$, where $\beta_{E(0)}$ is defined as in (2.9) and the function $\Theta$ is defined by

$$
\Theta(x)=\frac{e^{-\frac{1}{x}}}{x^{2}} \quad x>0 .
$$

Moreover the following property holds

$$
\lim _{t \rightarrow \infty}\left(\frac{\Theta^{-1}\left(\frac{1}{t}\right)}{g^{-1}\left(\frac{1}{t}\right)}\right)=1 .
$$

so that the energy satisfies the estimate

$$
E(t) \leq \beta_{E(0)}(\ln (t))^{-2}
$$

Example 3: let $g$ be given by $g(x)=x^{p}\left(\ln \left(\frac{1}{x}\right)\right)^{q}$ where $p>2$ and $q>1$ on $\left(0, r_{0}\right]$.

Then the energy of solution of (3.1) satisfies the estimate

$$
E(t) \leq \beta_{E(0)}\left(\Theta^{-1}\left(\frac{D}{t}\right)\right)^{2}
$$

for $t$ sufficiently large and for all $\left(u_{0}, u_{1}\right) \in H_{0}^{1}(\Omega) \times L^{2}(\Omega)$, where $\beta_{E(0)}$ is defined as in (2.9) and the function $\Theta$ is defined by 


$$
\Theta(x)=x^{p-1}\left(\ln \left(\frac{1}{x}\right)\right)^{q} \quad x \in(0,1)
$$

Example 4: let $g$ be given by $g(x)=e^{-\left(\ln \left(\frac{1}{x}\right)\right)^{p}}$ where $1<p<2$ on $\left(0, r_{0}\right]$.

Then the energy of solution of (3.1) satisfies the estimate

$$
E(t) \leq \beta_{E(0)}\left(\Theta^{-1}\left(\frac{D}{t}\right)\right)^{2}
$$

for $t$ sufficiently large and for all $\left(u_{0}, u_{1}\right) \in H_{0}^{1}(\Omega) \times L^{2}(\Omega)$, where $\beta_{E(0)}$ is defined as in (2.9) and the function $\Theta$ is defined by

$$
\Theta(x)=x^{-1}\left(\ln \left(\frac{1}{x}\right)\right)^{p-1} e^{-\left(\ln \left(\frac{1}{x}\right)\right)^{p}} \quad x \in(0,1) .
$$

Example 5: let $g$ be given by $g(x)=x\left(\ln \left(\frac{1}{x}\right)\right)^{-p}$ where $p>0$.

Then the energy of solution of (3.1) satisfies the estimate

$$
E(t) \leq p \beta_{E(0)} e^{-2\left(\frac{t}{D}\right)^{1 /(p+1)}}\left(\frac{D}{t}\right)^{1 /(p+1)}
$$

for $t$ sufficiently large and for all $\left(u_{0}, u_{1}\right) \in H_{0}^{1}(\Omega) \times L^{2}(\Omega)$ and where $\beta_{E(0)}$ is defined as in (2.9).

Remark: Only very few results are available on optimality. We refer to [6], [18] and [17] and the references therein for such results. Using Vancostenoble and Martinez results [17], we can show that the results for the four fi rst above examples for a one-dimensional wave equation with a boundary dissipation term, give optimal energy decay rates.

\section{Links between convexity and dissipation: some main ideas}

Two main different steps in nature are required for suitable energy estimates which can lead to energy decay rates. One is concerned with the estimate of a suitable weighted $L^{1}$-norm of the energy in terms of the linear and nonlinear kinetic energies. This step is proved in general using multiplier types methods eventually together with sharp trace regularity techniques. At the end of this step, an estimate of the form (2.11) is in general obtained. And that is for this step that geometric assumptions on the active region are required. The next step is to deduce from such an inequality, energy decay rates. This requires to obtain suitable "control" of the linear and nonlinear kinetic energies. This is where the link between convexity properties of the function $H$ introduced in (2.5) and the dissipation relation (2.4) is essential. The understanding of this link allows us to choose the "optimal" weight function, optimal in the sense that it leads to precise decay rates (shown to be optimal for several examples).

\section{Step 1: Estimate of the nonlinear kinetic energy:}

The nonlinear kinetic energy is given by: 


$$
\int_{S}^{T} f(E(t)) \int_{\Omega}\left|\rho\left(x, u^{\prime}(t)(x)\right)\right|^{2} d x d t
$$

To estimate this energy, we introduce for all fi xed $t \geq 0$, a partition $\left\{\Omega_{1}^{t}, \Omega \backslash \Omega_{1}^{t}\right\}$, of $\Omega$, where $\Omega_{1}^{t}=\left\{x \in \Omega,\left|u^{\prime}(t)(x)\right| \leq \varepsilon_{0}\right\}$ and $\varepsilon_{0}=g\left(r_{0}\right)$. One can prove, that there exists a known constant $c_{g}>0$ such that

$$
\frac{1}{\left|\Omega_{1}^{t}\right|} \int_{\Omega_{1}^{t}} c_{g}^{2}\left|\rho\left(x, u^{\prime}(t)(x)\right)\right|^{2} d x \in\left[0, r_{0}^{2}\right]
$$

which is the domain of convexity of $H$, and thanks to Jensen's inequality, we have

$$
H\left(\frac{1}{\left|\Omega_{1}^{t}\right|} \int_{\Omega_{1}^{t}} c_{g}^{2}\left|\rho\left(x, u^{\prime}(t)(x)\right)\right|^{2} d x\right) \leq \frac{1}{\left|\Omega_{1}^{t}\right|} \int_{\Omega_{1}^{t}} c_{g} \mid \rho\left(x, u^{\prime}(t)(x) \mid g\left(c_{g}\left|\rho\left(x, u^{\prime}(t)(x)\right)\right|\right) d x .\right.
$$

But, thanks to $(H F)$ and (5.1), one can prove

$$
H\left(\frac{1}{\left|\Omega_{1}^{t}\right|} \int_{\Omega_{1}^{t}} c_{g}^{2}\left|\rho\left(x, u^{\prime}(t)(x)\right)\right|^{2} d x\right) \leq \frac{1}{\left|\Omega_{1}^{t}\right|} \int_{\Omega_{1}^{t}} c_{g} u^{\prime}(t)(x) \rho\left(x, u^{\prime}(t)(x)\right) d x .
$$

On the other hand, one can also prove that

$$
H^{-1}\left(\frac{1}{\left|\Omega_{1}^{t}\right|} \int_{\Omega_{1}^{t}} c_{g} u^{\prime}(t)(x) \rho\left(x, u^{\prime}(t)(x)\right) d x\right) \in\left[0, r_{0}^{2}\right] .
$$

Let now $f$ be an arbitrary function in the set $\mathcal{F}_{\beta_{E(0)}, r_{0}^{2}}$ defi ned by (2.10) where $\beta=\beta_{E(0)}$ will be specifi ed later. Then, thanks to (5.2), we obtain that

$$
\begin{array}{r}
\int_{S}^{T} f(E(t)) \int_{\Omega_{1}^{t}}\left|\rho\left(x, u^{\prime}(t)(x)\right)\right|^{2} d x d t \leq \\
\int_{S}^{T} \frac{\left|\Omega_{1}^{t}\right|}{c_{g}^{2}} f(E(t)) H^{-1}\left(\frac{1}{\left|\Omega_{1}^{t}\right|} \int_{\Omega_{1}^{t}} c_{g} u^{\prime}(t)(x) \rho\left(x, u^{\prime}(t)(x)\right) d x\right) d t
\end{array}
$$

We defi ne now $\widehat{H}$ as in (2.6). Then, $\widehat{H}$ is a convex and proper function. Hence, we can apply Young's inequality (see [19],[16]) to any numbers $A$ and $B$ in $\mathbb{R}$, that is

$$
A B \leq \widehat{H}^{\star}(A)+\widehat{H}(B) .
$$

We apply the above inequality to $A=A(t)=f(E(t))$ and

$$
B=B(t)=H^{-1}\left(\frac{1}{\left|\Omega_{1}^{t}\right|} \int_{\Omega_{1}^{t}} c_{g} u^{\prime}(t)(x) \rho\left(x, u^{\prime}(t)(x)\right) d x\right) .
$$

Since $B(t) \in\left[0, r_{0}^{2}\right]$, and thanks to the dissipation relation (2.4), we deduce that

$$
\begin{array}{r}
\frac{\left|\Omega_{1}^{t}\right|}{c_{g}^{2}} f(E(t)) H^{-1}\left(\frac{1}{\left|\Omega_{1}^{t}\right|} \int_{\Omega_{1}^{t}} c_{g} u^{\prime}(t)(x) \rho\left(x, u^{\prime}(t)(x)\right) d x \leq\right. \\
\frac{\left|\Omega_{1}^{t}\right|}{c_{g}^{2}} \widehat{H}^{\star}(f(E(t)))+\frac{1}{c_{g}} \int_{\Omega_{1}^{t}} u^{\prime}(t)(x) \rho\left(x, u^{\prime}(t)(x)\right) d x \leq \\
\frac{|\Omega|}{c_{g}^{2}} \widehat{H}^{\star}(f(E(t)))+\frac{1}{c_{g}}\left(-E^{\prime}(t)\right)
\end{array}
$$


We use (5.6) in (5.4).

$$
\int_{S}^{T} f(E(t)) \int_{\Omega_{1}^{t}}\left|\rho\left(x, u^{\prime}(t)(x)\right)\right|^{2} d x d t \leq \frac{|\Omega|}{c_{g}^{2}} \int_{S}^{T} \widehat{H}^{\star}(f(E(t))) d t+\frac{1}{c_{g}} E(S), \quad \forall 0 \leq S \leq T .
$$

On $\Omega \backslash \Omega_{1}^{t}$, we use the fact that $\rho$ has a linear growth with respect to the second variable. Hence, one can prove that

$$
\int_{S}^{T} f(E(t)) \int_{\Omega-\Omega_{1}^{t}}\left|\rho\left(x, u^{\prime}(t)(x)\right)\right|^{2} d x d t \leq \frac{1}{c_{g}} E(S) f(E(S)) .
$$

Both estimates (5.7) and (5.8) lead to the following estimate of the nonlinear kinetic energy for all $0 \leq S \leq T$

$$
\int_{S}^{T} f(E(t)) \int_{\Omega_{1}^{t}}\left|\rho\left(x, u^{\prime}(t)(x)\right)\right|^{2} d x d t \leq \frac{|\Omega|}{c_{g}^{2}} \int_{S}^{T} \widehat{H}^{\star}(f(E(t))) d t+\frac{1}{c_{g}} E(S)+\frac{1}{c_{g}} E(S) f(E(S)) .
$$

\section{Estimate of the linear kinetic energy on the active region:}

The linear kinetic energy on the active region is defi ned by:

$$
\int_{S}^{T} f(E(t)) \int_{\omega}\left|u^{\prime}(t)(x)\right|^{2} d x d t
$$

To estimate this energy, we introduce for all fi xed $t \geq 0$, a partition $\left\{\omega_{1}^{t}, \omega \backslash \omega_{1}^{t}\right\}$, of $\omega$, where $\omega_{1}^{t}=\left\{x \in \omega,\left|u^{\prime}(t)(x)\right| \leq \varepsilon_{1}\right\}$ and $\varepsilon_{1}=\min \left(r_{0}, g\left(r_{1}\right)\right)$, with $r_{1}^{2}=H^{-1}\left(c_{1} a_{-} c_{g} H\left(r_{0}^{2}\right)\right)$.

Using $(H F)$, the dissipation relation and proceeding in a similar way than for the nonlinear kinetic energy, one can prove that

$$
\int_{S}^{T} f(E(t)) \int_{\omega_{1}^{t}}\left|u^{\prime}(t)(x)\right|^{2} d x d t \leq|\omega| \int_{S}^{T} \widehat{H}^{\star}(f(E(t))) d t+c E(S), \quad \forall 0 \leq S \leq T .
$$

On $\omega-\omega_{1}^{t}$, we use once again the linear growth of $\rho$. This implies

$$
\int_{S}^{T} f(E(t)) \int_{\omega-\omega_{1}^{t}}\left|u^{\prime}(t)(x)\right|^{2} d x d t \leq c E(S) f(E(S)) .
$$

Using these two last inequalities, we obtain the following estimate of the linear kinetic energy on the active region

$$
\begin{array}{r}
\int_{S}^{T} f(E(t)) \int_{\omega}\left|u^{\prime}(t)(x)\right|^{2} d x d t \leq|\omega| \int_{S}^{T} \widehat{H}^{\star}(f(E(t))) d t+c E(S)+ \\
c E(S) f(E(S)), \quad \forall 0 \leq S \leq T .
\end{array}
$$

Inserting now (5.9), and (5.12) in (2.11), we obtain

$$
\begin{gathered}
\int_{S}^{T} f(E(t)) E(t) d t \leq\left(\delta_{1}+\frac{\delta_{2}}{c_{g}}+c \delta_{3}\right) E(S) f(E(S))+\left(\frac{\delta_{2}}{c_{g}}+c \delta_{3} E(S)+\right. \\
\left(\delta_{2} \frac{|\Omega|}{c_{g}^{2}}+\delta_{3}|\omega|\right) \int_{S}^{T} \widehat{H}^{\star}(f(E(t))) d t .
\end{gathered}
$$


We defi ne $F$ by (2.7). We recall that $F$ is a strictly increasing function from $[0,+\infty)$ onto $\left[0, r_{0}^{2}\right)$. We also choose a real number $\beta=\beta_{E(0)}$ as follows:

$$
\beta=\max \left(\delta_{3}|\omega|+\frac{\delta_{2}|\Omega|}{c_{g}^{2}}, \frac{E(0)}{2 F\left(H^{\prime}\left(r_{0}^{2}\right)\right)}\right)
$$

Now, we choose the weight function $f$ as announced, that is:

$$
f(s)=F^{-1}\left(\frac{s}{2 \beta}\right) \quad \forall s \in\left[0,2 \beta r_{0}^{2}\right) .
$$

Then $f$ is a strictly increasing function from $\left[0,2 \beta r_{0}^{2}\right)$ onto $[0,+\infty)$. Moreover $f$ satisfi es the relation:

$$
\beta \widehat{H}^{\star}(f(s))=\frac{1}{2} s f(s) \quad \forall s \in\left[0,2 \beta r_{0}^{2}\right) .
$$

Since $E$ is nonincreasing, we have

$$
E(t) \leq E(0)<E(0) \frac{r_{0}^{2}}{F\left(H^{\prime}\left(r_{0}^{2}\right)\right)} \leq 2 \beta r_{0}^{2} \quad \forall t \geq 0 .
$$

Hence, one has in particular:

$$
\beta \widehat{H}^{\star}(f(E(t)))=\frac{1}{2} E(t) f(E(t)) \quad \forall t \geq 0 .
$$

With this choice of $\beta$ and $f$, the last term on the right hand side of (5.13) is bounded above by

$$
\frac{1}{2} \int_{S}^{T} E(t)(f(E(t))) d t
$$

We insert the estimate (5.17) in (5.13). This gives

$$
\int_{S}^{T} E(t) F^{-1}\left(\frac{E(t)}{2 \beta}\right) d t \leq 2\left(\delta_{1}+\frac{\delta_{2}}{c_{g}}+c \delta_{3}\right) E(S) F^{-1}\left(\frac{E(S)}{2 \beta}\right)+2\left(\frac{\delta_{2}}{c_{g}}+c \delta_{3} E(S) .\right.
$$

Hence, the energy $E$ satisfi es the estimate

$$
\int_{S}^{T} E(t) F^{-1}\left(\frac{E(t)}{2 \beta}\right) d t \leq T_{0} E(S) \quad \forall 0 \leq S \leq T,
$$

where $T_{0}$ is independent of $E(0)$ and, with our choice of $\beta$ is given by

$$
T_{0}=2\left(\frac{\delta_{2}}{c_{g}}+c \delta_{3}+\left(\delta_{1}+\frac{\delta_{2}}{c_{g}}+c \delta_{3}\right) H^{\prime}\left(r_{0}^{2}\right)\right)
$$

Once, the above weighted integral inequality is obtained for $E$, we can conclude by a fi nal step described shortly in the following result.

Theorem 7. Let $g$ be a given odd, strictly increasing $C^{1}$ function from $\mathbb{R}$ to $\mathbb{R}$ such that $g^{\prime}(0)=0$. We assume that there exists $r_{0}>0$ such that $g$ is of class $C^{2}$ on $\left[0, r_{0}\right]$ and the function $H$ defined by (2.5) is strictly convex on $\left[0, r_{0}^{2}\right]$. We define $\widehat{H}$ and $F$ by (2.6) and (2.7) respectively. Moreover, 
let $T_{0}>0$ be a fixed real number, $E$ be a given nonincreasing, absolutely continuous, nonnegative real function defined on $[0,+\infty)$, and $\beta>0$ a given real number such that

$$
0<\frac{E(0)}{2 F\left(H^{\prime}\left(r_{0}^{2}\right)\right)} \leq \beta
$$

and,

$$
\int_{S}^{T} E(t) F^{-1}\left(\frac{E(t)}{2 \beta}\right) d t \leq T_{0} E(S), \quad \forall 0 \leq S \leq T .
$$

Then, E decays at infinity as follows:

$$
E(t) \leq 2 \beta z^{2}(t) \frac{z(t) g^{\prime}(z(t))-g(z(t))}{z(t) g^{\prime}(z(t))+g(z(t))}, \quad \forall t \geq \frac{T_{0}}{H^{\prime}\left(r_{0}^{2}\right)},
$$

where $z, \phi$ and $\alpha$ are respectively defined as in (2.13), (2.14) and (2.15).

\section{References}

[1] F. Alabau-Boussouira, Convexity and weighted integral inequalities for energy decay rates of nonlinear dissipative hyperbolic systems. Appl. Math. and Optimization 51, no. 1, 61-105 (2005).

[2] F. Alabau-Boussouira, Une formule générale pour le taux de décroissance des systèmes dissipatifs non linéaires. C. R. Acad. Sci. Paris Sér I Math, 338: 35-40 (2004).

[3] C. Bardos, G. LebeaU, J. RAUCh Sharp suffi cient conditions for the observation, control, and stabilization of waves from the boundary. SIAM J. Control Optimization 30, 1024-1065 (1992).

[4] F. BUCCI, Uniform decay rates of solutions to a system of coupled PDE's with nonlinear internal dissipation. Diff. and Integral Equations 16, 865-896 (2003).

[5] M. M. Cavalcanti, V. N. Cavalcanti, P. Martinez Existence and decay rates estimates for the wave equation with nonlinear boundary damping and source term. To appear in J. of Diff. Equations.

[6] A. HARAUX, Nonlinear evolution equations—global behavior of solutions. Lecture Notes in Mathematics 841, Springer-Verlag Berlin-NewYork (1981).

[7] A. Haraux, E. Zuazua Decay estimates for some semilinear damped hyperbolic problems. Arch. Rational Mech. Anal. 100, 191-206 (1988).

[8] V. Komornik, Exact Controllability and Stabilization. The Multiplier Method. Collection RMA, vol. 36, Masson-John Wiley, Paris-Chicester, 1994.

[9] I. LASIECKA, D. TATARU Uniform boundary stabilization of semilinear wave equation with nonlinear boundary damping. Differential and Integral Equations 8, 507-533 (1993).

[10] I. LASIECKA, Mathematical Control Theory of coupled PDE's. CBMS-NSF Regional Conference Series in Applied Math 75, SIAM (2002).

[11] K. LIU, Locally distributed control and damping for the conservative systems. SIAM J. Control Optimization 35, 1574-1590 (1997).

[12] W.-J. LiU AND E. ZuAzuA, Deacy rates for dissi pative wave equations. Ricerche di Matematica, 48, 61-75 (1999). 
[13] P. MARTINEZ, A new method to obtain decay rate estimates for dissipative systems with localized damping. Rev. Mat. Complut. 12, 251-283 (1999).

[14] P. MARTINEZ, A new method to obtain decay rate estimates for dissipative systems. ESAIM Control Optim. Calc. Var. 4 (1999), 419-444 (1999).

[15] M. NAKAO, Decay of solutions of the wave equation with a local nonlinear dissipation. Math. Ann. 305, 403-417 (1996).

[16] R. T. Rockafellar, Convex analysis. Princeton University Press, 1970.

[17] J. VANCOSTENOBLE AND P. MARTINEZ, Optimality of energy estimates for the wave equation with nonlinear boundary velocity feedbacks. SIAM J. Control Optimization 39, 776-797 (2000).

[18] J. Vancostenoble, Optimalité d'estimation d'énergie pour une équation des ondes amortie. C. R. Acad. Sci. Paris, 328, série I, 777-782 (1999).

[19] W. H. YounG, On classes of summable functions and their Fourier series. Proc. Royal Soc. (A) 87, 225-229 (1912).

[20] E. ZUAZUA, Exponential decay for the semilinear wave equation with locally distributed damping. Comm. in P.D.E. 15, 205-235 (1990).

[21] E. ZUAZUA, Uniform stabilization of the wave equation by nonlinear feedbacks. SIAM J. Control Optimization 28, 265-268 (1989). 